\title{
A computational view of sea urchin development
}

As an embryo develops into an adult, each cell follows a genetic 'script' comprising an intricate network of interactions between regulatory genes. Extensive research has been done to identify these genes and their interactions in order to build a more detailed understanding of how development progresses. Sea urchin embryos are favored models for such research, owing to their manageable size and their transparency, which enables direct visualization of developmental stages.

Biologists at the California Institute of Technology (Pasadena) have focused their research on characterizing the gene interactions that occur during the first 30 hours of development of the endomesoderm in the sea urchin. They have identified $\sim 50$ regulatory genes in this network. Now, for the first time, they have built a computational model of this network, creating a powerful tool for exploring gene regulatory networks (GRNs) in a novel manner.

To construct the model, Isabelle S. Peter, Emmanuel Faure and Eric H. Davidson condensed everything they had learned about the network into a series of logical 'if-then' (or Boolean) statements (e.g., if gene 1 is on, then gene 2 is turned off). By analyzing these sequential statements, the model predicts the location and timing of expression of each gene within the developing embryo (Proc. Natl. Acad. Sci. USA published online 27 August 2012; doi:10.1073/pnas.1207852109). The group compared the predicted expression patterns with experimental observations and found that the model reproduced the data very closely. "It works surprisingly well," said Peter in a press release.

The team also introduced alterations into the model, such as deactivating or misexpressing certain genes, manipulations that are often done in vivo to assess their effects on development. The model correctly predicted the same results that are seen experimentally in response to such manipulations.

Peter and her team draw two key conclusions from the success of their computational GRN model. First, their current understanding of the network controlling sea urchin endomesoderm

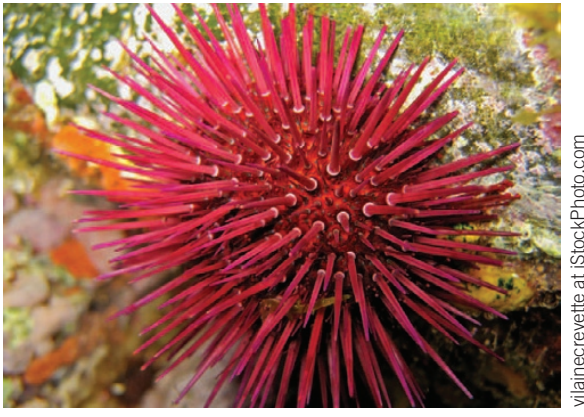

development during the first 30 hours is both thorough and accurate. There were a few inaccuracies in the model's predictions, but these helped to identify pockets of missing or incomplete data. "Identification of these gaps in the GRN model is one of the useful outcomes of the computation," the authors wrote. Second, their computational approach accurately represents developmental regulation and can be used to test developmental perturbations in silico. Their goal is to similarly model the GRNs underlying each part of a sea urchin embryo during its entire development.

Monica Harrington

\section{ROBOT ALLOWS NEUROSCIENTISTS TO ACCESS BRAIN CELLS}

Technology that can find and simultaneously record the activity of dozens of neurons in live animals could help researchers to reveal how connected cells interpret signals from one another and transmit information across brain areas. Craig R. Forest (Georgia Institute of Technology, Atlanta) and collaborators at Massachusetts Institute of Technology in Cambridge have now developed such a technology by constructing a robot that can perform in vivo whole-cell patch-clamping of neurons (Nat. Methods published online 6 May 2012 ; doi:10.1038/nmeth.1993).

Whole-cell patch-clamping is a method used by neuroscientists to measure electrical currents inside neurons. These electrical currents can reveal information about how the functioning of neurons is affected by brain disorders or by drug treatment. The technique also allows scientists to access brain cells in order to infuse dyes or to extract cell contents for gene expression analysis. Manually patch-clamping cells in live animals requires extensive training to perfect, and only a handful of neurophysiologists are trained to perform the technique.

Forest and his team first created an algorithm to reduce the technique for whole-cell path-clamping of neurons to four steps. In the first step, the pipette is rapidly lowered to the desired depth. Next, the pipette is advanced more slowly until a neuron is detected. Then, suction is applied to create a seal between the pipette and the surface of the cell. Finally, a brief voltage pulse is applied to allow the pipette to 'break in' to the cell.

The group next created a robot that could perform this algorithm in about 5 minutes, similar to the time it would take an experienced investigator to perform the technique. The robot could be used to record electrical currents from single neurons in the brains of anesthetized mice. While the algorithm was derived from patching of cortical cells, the researchers showed that the robot could be used in the hippocampus as well. Using the robot, the researchers were able to obtain successful whole-cell patch recordings $32.9 \%$ of the time, similar to the success rate of a trained investigator manually performing the technique. When a histological stain was included in the pipette solution, cell morphologies could be visualized histologically.

Currently, the robot can identify and probe up to four neurons at a time, though the goal is to further increase that number. Simultaneous recordings from multiple cells will allow neuroscientists to learn about how the cells communicate with each other to affect cognition and behavior. 